\title{
CAPITAL PÚBLICO Y PRODUCTIVIDAD REGIONAL: EVIDENCIA EMPÍRICA PARA ESPAÑA
}

\author{
Sergio Jiménez Ramírez* \\ Jaime Sanaú Villarroya ${ }^{* *}$
}

\footnotetext{
* Ph.D. En Economía Universidad de Zaragoza (España).Profesor Departamento de Economía, Universidad de Pamplona (Colombia).

** Profesor Departamento de Estructura e Historia Económica y Economía Pública, Universidad de Zaragoza (España).
} 


\section{CAPITAL PÚBLICO Y PRODUCTIVIDAD REGIONAL: EVIDENCIA EMPÍRICA PARA ESPAÑA}

\section{RESUMEN}

En el presente artículo se realiza un análisis empírico sobre el efecto del capital público en la producción agregada regional, aplicado a las 17 comunidades autónomas españolas durante el periodo 1988-2001, observando la presencia de spillovers. Además, se amplía el análisis habitual en otros trabajos estudiando el efecto de diferentes clases o categorías de capital público sobre la economía agregada regional y analizando sus efectos sobre los más importantes sectores de la activad económica. En la función de producción agregada regional -además de trabajo, capital privado y capital público- se incluyen el capital humano y el capital tecnológico, omitidos en buena parte de la literatura previa. Se usan datos oficiales del INE así como los últimos publicados por la Fundación BBVA y el IVIE sobre capitales físico privado y público. Los resultados confirman una notable contribución del capital público a la producción privada regional, especialmente sobre los sectores de industria y servicios disponibles para la venta, y como categorías más importantes las de carreteras y autopistas, infraestructuras hidráulicas, aeropuertos y puertos.

Palabras Claves: productividad regional, crecimiento, categorías del capital público, capital humano.

\begin{abstract}
In this paper, we present an empirical analysis on the effect of public capital in regional aggregate output, applied to the 17 Spanish autonomous communities during the period 1988-2001, noting the presence of spillovers. Furthermore, it extends the usual analysis in other works studying the effect of different classes or categories of public capital on aggregate regional economy and analyze its effects on the most important sectors of economic activity. In the aggregate production function also working regionally, private capital and public capital includes human capital and technological capital, missing in much of the previous literature. They are used official data from the INE and the latest published by the BBVA Foundation and the Ivie on private and public physical capital. Our results confirm the significant contribution of public capital to private production regionally, especially on the industrial and services available for sale, and as most important categories of roads and highways, water infrastructure, airports and ports.
\end{abstract}

Keywords: regional productivity growth, categories of public capital, human capital.

\section{Face ISSN 1794-9920}

Recepción: Julio de 2008

Revisión: Diciembre de 2008

Aceptación: Diciembre de 2008 


\section{INTRODUCCIÓN}

Hace ya varias décadas surgió entre los economistas la idea de que la inversión pública en infraestructuras (o capital público, como se conoce en la literatura especializada), ayuda a incrementar la productividad, y ya para los años cincuenta de la pasada centuria algunos autores comenzaron a referirse formalmente al tema ${ }^{1}$. Sin embargo, fue una motivación de más peso, como la desaceleración del crecimiento de la productividad ocurrida en la década de 1970 en Estados Unidos, tras mantener un elevado crecimiento en los sesenta, la que renovaría el interés de los investigadores por los factores que dinamizan la productividad y el crecimiento económico.

En un trabajo seminal publicado en 1989 David Aschauer presentó unos resultados que otorgaban al capital público el rol de factor relevante en el proceso de producción con una elasticidad del producto con respecto a esta variable de 0,39. A partir de este trabajo se desencadenó una copiosa literatura, con una notoria variedad de resultados. Muchos de estos nuevos estudios, incluidos varios aplicados a España, apoyaron los resultados de Aschauer, aunque obtuvieron un valor para la elasticidad quizá más creíble. Otros, no obstante con similar grado de aprobación científica desmintieron la hipótesis de que el capital público sea un factor de producción adicional tan importante como se entendía a partir del seminal artículo.

De los principales estudios realizados para España puede concluirse, en términos generales, que el capital público y las infraestructuras tienen un efecto positivo y apreciable sobre la productividad y el crecimiento de su economía. Algunos autores, entre ellos Fernández y Polo (2002), Álvarez et al. (2003) y Pérez y Sanaú (2004), han resaltado la disparidad en los resultados atribuyendo diversas causas posibles. En nuestra opinión este hecho puede ser producto de diferencias en la utilización de metodologías y técnicas econométricas, la definición del capital público, las fuentes de datos utilizadas y las variables explicativas incluidas. De esta relativa incertidumbre que aún subsiste han surgido las razones que motivan este trabajo. Pese a que está claro que en los últimos años se han ido despejando las dudas acerca de los elementos básicos de la relación entre capital público y crecimiento económico, creemos que se puede aportar mayor robustez a las hipótesis fundamentales, así como profundizar en relaciones aún no lo suficientemente claras.

Con este propósito, esta investigación pretende estudiar, en primer lugar, el efecto del capital público en la producción regional (las 17 comunidades autónomas españolas), desagregándolo en diferentes clases o categorías. En segundo lugar, los efectos en algunos de los más importantes sectores de la economía. Y todo ello, en presencia de dos variables adicionales, como son el capital humano y el capital tecnológico, que la

1 Véase Meade (1952) o Hirschman (1958), por ejemplo. 
literatura previa tendía a omitir. En este punto se pretende contrastar los resultados de Fernández y Polo (2001 y 2002) quienes sostienen que las elevadas productividades del capital público a nivel agregado se reducen o incluso se desvanecen al incluir otras variables que afectan la productividad total de los factores. Finalmente, se aborda un periodo muestral más reciente, 1988-2001, con los últimos datos a este respecto publicados por la Fundación BBVA y el IVIE².

Debe destacarse que en este trabajo se abordan cuatro elementos nuevos en el análisis convencional a escala regional, como son, en primer lugar, la desagregación del capital público en diferentes categorías, en segundo lugar, la desagregación de la economía en sus sectores más importantes, en tercer lugar, una mejor especificación del modelo al incluir dos nuevas variables: el capital humano y el capital tecnológico, y por último, un periodo más reciente (aún sin explorar) con mejores datos y fuentes. Se han planteado cinco objetivos puntuales que permitirán con su desarrollo aportar elementos nuevos que puedan despejar alguna de las incógnitas que aún persisten en la relación existente entre el capital público, la productividad y el crecimiento económico regional. Los objetivos son:

1) Observar el comportamiento del capital público dentro de la función de producción ampliada de Aschauer, en presencia de dos nuevas variables, capital humano $(K H)$ y capital tecnológico $(K T)$.

2) Comprobar la importancia de la composición del capital público, dividiéndolo en dos categorías, como son el capital público económico $(K G E)$ y el capital público social $(K G S)$.

3) Determinar qué subcategorías del capital público son más importantes para el crecimiento de la producción, así como contrastar el "efecto cooperación o sinérgico". 3

4) Evaluar si existen “efectos desbordamiento” o spillovers en el capital público.

5) Analizar que subsectores de la economía española son los más afectados en su productividad por el capital público total, económico y social, así como por alguna de las subcategorías de éste, además de evaluar el modelo conjuntamente para los dos sectores más importantes de la economía española, como son la industria y los servicios.

\footnotetext{
2 Se tomó este periodo de tiempo en vista de que los datos de gastos en I+D del INE sólo están disponibles a partir de 1987, y los datos de los stocks de capital privado y público de la Fundación BBVAIVIE sólo está disponibles hasta 2000, además de que la Contabilidad Regional del INE sólo ofrece datos desde 1986.

${ }^{3} \mathrm{El}$ "efecto cooperación o sinérgico" sugiere que los efectos en la actividad económica de un conjunto de infraestructuras suelen ser mayores que los de las diferentes subcategorías individualmente consideradas, tal como indica Sanaú (1995).
} 
En el epígrafe siguiente se realiza una breve revisión de la literatura empírica sobre la productividad del capital público poniendo especial énfasis en el caso español; en el apartado 3 se expone el modelo empírico, los datos, las fuentes y las variables utilizadas; en la 4 se estima el modelo y se muestran los principales resultados. Por último, en el epígrafe 5 se exponen las conclusiones más importantes.

\section{LA CONTRASTACIÒN DE LA HIPÓTESIS DEL CAPITAL PÚBLICO}

Aunque la idea de que las infraestructuras, y la inversión pública en este tipo de bienes, ayudan a incrementar la productividad ya se discutía en la década de 1950, el hecho de considerarlas como factor relevante para el crecimiento de la producción se constató a finales de la década de 1980 cuando Aschauer publicó su original trabajo. A partir de entonces, numerosos autores han intentado aportar evidencia empírica a la hipótesis de que el capital público cumple un importante papel en el proceso de producción y en los incrementos de productividad. Tanto en el ámbito nacional como internacional existen varias formas de aproximación a este tema haciendo uso de la función de producción agregada: la que emplea series temporales de variables agregadas para el conjunto de la economía y la que utiliza paneles de datos para las distintas regiones, estados o comunidades autónomas. Existe otro tipo de aproximación, aunque menos común, que utiliza funciones de costes y de beneficios en vez de una función de producción. ${ }^{4} \mathrm{~A}$ continuación se exponen muy brevemente dos de los trabajos más importantes y pioneros en este campo realizados siguiendo el primero de estos enfoques.

Aschauer (1989) motivado por determinar los posibles factores que generaron la desaceleración del crecimiento de la productividad en la década de 1970 en Estados Unidos tras mantener un elevado crecimiento en el decenio de 1960, indagó acerca de la relación entre la productividad agregada y variables de flujo y stock de gastos del gobierno, estimando mediante series temporales para la economía agregada una función de producción ampliada tipo Cobb-Douglas.

Los resultados de Aschauer indicaron que el stock de capital público no militar era mucho más importante como determinante de la productividad que los flujos de gasto no militar o militar. Además, encontró que dentro de este capital había infraestructuras clave que tenían mayor poder explicativo para las variaciones en la productividad, tales como carreteras, autopistas, aeropuertos, sistemas de acueductos y alcantarillados, entre otros. La elasticidad obtenida para el capital público fue cercana a 0,39. En suma, este autor concluyó que el stock de capital público neto pudo jugar un importante papel en la desaceleración de la productividad de la década de 1970 en los Estados Unidos.

\footnotetext{
${ }_{4}^{4}$ También se han empleado funciones de cuasi producción. Véase, por ejemplo, Biehl (1988).
} 
Munnell (1992) extendió el trabajo de Aschauer (1989) y desagregó el análisis al nivel regional utilizando datos de panel para los estados norteamericanos. En su estudio la elasticidad del capital público resultó de menor magnitud que para el conjunto de la economía, siendo casi la mitad del correspondiente al nivel federal. Esto se explicó, en parte, por la existencia de efectos desbordamiento de las infraestructuras o efectos externos interregionales.

Inspirados en Aschauer y Munnell, un buen número de estudios han continuado la investigación empírica de la relación entre capital público y output. En las siguientes líneas se realiza una breve revisión de la literatura, en la que se han seleccionado algunos de los trabajos realizados con aplicación a España que utilizan el enfoque de la función de producción ampliada Cobb-Douglas, así como los publicados en los últimos años.

Mas, Maudos, Pérez y Uriel (1996) analizaron los efectos del capital público sobre el Valor Añadido Bruto (VAB) y la productividad de las regiones españolas ampliando el análisis de estudios previos realizados por ellos mismos, pues en este trabajan el periodo es 1964-1991. Emplearon un modelo donde la variable endógena es el VAB y las explicativas el empleo, capital físico privado y capital público. Estos autores se centraron en tres puntos de interés para lo que llevaron a cabo las siguientes acciones.

En primer lugar, estimaron los efectos del capital público y privado sobre la productividad del trabajo a nivel regional, sin imponer restricciones sobre el tipo de rendimientos en la función de producción; en segundo lugar, analizaron la importancia de la composición (productivo/social) del stock de capital público; y por último, observaron si la influencia del capital público depende solamente de las infraestructuras instaladas en una región particular o también de las dotaciones de las regiones vecinas.

En referencia al primero de los objetivos, ellos obtuvieron una elasticidad del VAB con respecto al capital público positiva y significativa $(0,071)$. En cuanto al análisis de la composición del capital público, confirmaron su hipótesis obteniendo una elasticidad de la productividad del trabajo con respecto al capital público de carácter productivo de 0,083, mientras que en el caso del capital público de carácter social fue negativa aunque no significativa. Asimismo, en el contraste del "efecto desbordamiento" o "efecto red" hallaron que el crecimiento en la elasticidad de la productividad con respecto al capital público de 0,071 a 0,141 cuando son tenidos en cuenta ambos, el capital público de cada región y el de las regiones adyacentes, es un indicador favorable para la aceptación de la hipótesis del efecto desbordamiento, lo que podría reflejar externalidades en este tipo de capital público. Un último hallazgo que se desprende del anterior señala que el capital público tiene diferentes efectos sobre la productividad dependiendo del nivel de desarrollo del país o región y del tamaño del stock de capital acumulado previamente. 
Freire-Serén y Alonso (2002) analizaron las posiciones relativas de las regiones españolas en cuanto a las dotaciones de infraestructuras públicas, con especial referencia a Galicia. Para ello estimaron la elasticidad de la renta regional por ocupado con respecto al stock de infraestructuras públicas. En tal sentido, estudiaron el periodo 1964-1993, considerando los años impares excepto en la primera observación que tomaron el año 1964 en lugar de 1965. La variable dependiente empleada fue el logaritmo del producto por ocupado en millones de pesetas de 1990 (aproximada por el ratio $\mathrm{VAB}$ por población ocupada). Las variables independientes empleadas fueron el stock neto de capital privado y el stock neto de capital público productivo regionales a precios constantes de 1990, capital humano aproximado por el porcentaje regional de ocupados con al menos estudios medios terminados y una variable que tenía en cuenta la superficie de cada comunidad autónoma.

Los resultados que Freire-Serén y Alonso obtuvieron de la estimación de la ecuación en niveles por mínimos cuadrados ordinarios con datos de panel a nivel regional respaldan la hipótesis de que las infraestructuras tienen un impacto positivo y significativo sobre la productividad regional en España. Además sus resultados también confirmaron la importancia de controlar por la extensión del territorio, pues la superficie presenta un efecto negativo y significativo sobre la productividad. Sus estimaciones proporcionaron una elasticidad del producto con respecto a las infraestructuras públicas del 0,126 y con respecto la superficie del -0,056. De ahí que concluyeran, que el stock de infraestructuras es una variable relevante para explicar las disparidades regionales observadas en la renta por ocupado, y establecen que el nivel observado de inversión pública en infraestructuras parecía situarse por debajo de su nivel óptimo en todas las regiones.

Fernández y Polo (2002) analizaron el impacto de las Administraciones Públicas en la productividad y en el proceso de crecimiento de la economía española entre 1964 y 1991. Emplearon un modelo donde la variable endógena era el VAB y las exógenas el empleo, el capital físico privado, el capital público, el capital humano y el capital tecnológico financiado con fondos públicos 5 . Su hipótesis de trabajo sugería que el efecto de las infraestructuras públicas sobre la productividad privada, aunque positivo, es sensible a la introducción de otras variables como los capitales humano y tecnológico. Según estos autores, los resultados obtenidos en los trabajos previos eran insatisfactorios en varios aspectos. Primero, los valores estimados en algunos casos para los factores privados (trabajo o capital) eran muy bajos. Segundo, existen diferencias muy notables entre los valores estimados por varios autores para el coeficiente del capital público que van desde un mínimo de 0,18 en Bajo y Sosvilla (1993) hasta 0,59 en Arguimón et al. (1994). Tercero, en muchos casos estos valores implican que la

\footnotetext{
${ }^{5}$ Aunque este trabajo se realiza empleando series temporales a nivel de la economía agregado nacional y no para las comunidades autónomas, nos resulta relevante pues es el primero que incluye en la función de producción ampliada de Aschauer el capital humano y el capital tecnológico de forma conjunta.
} 
productividad del capital público es demasiado elevada como para ser creíble. Cuarto, los valores de las elasticidades del capital público se reducen drásticamente cuando se introduce otra variable que representa al resto del capital público o una tendencia exógena.

A partir de la evidencia empírica disponible (la cual consideraron poco satisfactoria), enunciaron dos conclusiones preliminares. Primero, que la elasticidad de la producción privada respecto al capital público es más probable que se encuentre en el intervalo 0,15 - 0,30 que en el intervalo 0,50 - 0,60. Segundo, que los cambios en los valores estimados cuando se modifica la especificación sugieren que es posible que la omisión de alguna variable relevante haya podido sesgar al alza los coeficientes del capital público tangible. Los resultados obtenidos por Fernández y Polo apoyaron su hipótesis y sus dos conclusiones preliminares, pues las elevadas productividades del capital público mostradas en estudios previos se redujeron al incluir dichas variables en el modelo estimado, siendo en algunos casos no significativos los coeficientes estimados.

Álvarez, Orea y Fernández (2003) llevaron a cabo una importante revisión de los trabajos realizados en España junto con un análisis empírico sobre la productividad del capital público, con datos de las comunidades autónomas del periodo 1980-1995. En la función de producción agregada incluyeron variables como el capital humano o la especialización productiva regional. También, analizaron el denominado efecto desbordamiento del capital público, aunque a diferencia de otros trabajos, examinando las productividades marginales en vez de las elasticidades. El modelo básico que estimaron se basa en la especificación de Aschauer (1989). Para la estimación del modelo empírico utilizaron técnicas de datos de panel. A la serie de capital público del IVIE le restaron el capital público sanitario y educativo con el fin de lograr una definición de capital público productivo relativamente comparable con otros trabajos. Como variables explicativas utilizaron el stock de capital privado efectivo ajustado por el índice de utilización de la capacidad productiva, además del trabajo, el stock de capital público del IVIE y el de Delgado y Álvarez, el índice de capital humano $(H)$ y el índice de especialización productiva (IE). Los modelos se estimaron usando el estimador intragrupos.

Concluyeron que los índices del IVIE y de Delgado y Álvarez (2001) no producen estimaciones muy diferentes entre sí, puesto que la elasticidad del capital público productivo se sitúa entre 0,08 y 0,14 . Asimismo, sus resultados parecen indicar que las infraestructuras son más productivas en las regiones más desarrolladas que en las regiones con un menor grado de desarrollo. Encontraron que los efectos desbordamiento del capital público son muy sensibles a la forma en que se modelizan, si bien su elasticidad aumentaba en los tres casos estudiados. Además, concluyeron que cuando el efecto desbordamiento se estudia mirando la productividad marginal del capital público en vez de la elasticidad de producción, en algunos casos el capital 
público localizado en una determinada región genera externalidades positivas sobre otras regiones, aunque su productividad marginal en la propia región disminuye.

Delgado y Álvarez (2004) estudiaron el efecto de algunas infraestructuras sobre los sectores productivos de la economía española, adoptando opciones como la incorporación de la eficiencia técnica en la función a estimar y la utilización de unidades físicas para medir el capital, distinguiendo entre las dotaciones de transportes, comunicaciones y energía. Para ello plantearon un modelo de frontera de producción estocástica con objeto -afirman- de evitar el sesgo derivado de asumir que todas las unidades económicas son eficientes, y analizar simultáneamente el papel de las infraestructuras como input productivo y como determinante de la eficiencia técnica. Además, como medida de cuantificación de este capital utilizaron unidades físicas como enfoque alternativo al habitual, en unidades monetarias.

La evidencia obtenida por estas autoras para la economía española se asemeja a la de otros trabajos, con una elasticidad positiva y significativa de 0,12. También comprobaron que la formación de capital en infraestructuras condiciona los niveles y la evolución de la eficiencia de las actividades productivas. A nivel sectorial, pese a ciertas limitaciones sobre la estimación de su impacto, encontraron que el efecto económico de las infraestructuras se localiza en la industria, los servicios destinados a la venta, y en menor medida, en la construcción. Asimismo, detectaron amplias desigualdades regionales en los equipamientos de transportes, comunicaciones y energía, situándose buena parte de las regiones españolas por debajo de la media nacional. La estimación del impacto desagregado de estas tres categorías de infraestructuras mostró que los equipamientos de comunicaciones aumentan la productividad privada y mejoran la eficiencia productiva de las actividades económicas; las infraestructuras de transporte resultaron ser también un factor productivo relevante para explicar la productividad, aunque no condicionen las mejoras de eficiencia experimentadas por el conjunto de la economía española.

En síntesis, la evidencia empírica de la contrastación de la hipótesis del capital público para el caso de España resulta favorable, tanto a nivel agregado como regional. Con objeto de aportar evidencia adicional a dicha hipótesis, en el siguiente apartado se expone un modelo empírico que se estimará para un periodo temporal más reciente, en presencia de dos nuevos factores: el capital humano y el capital tecnológico. 


\section{MODELO EMPÍRICO, DATOS Y FUENTES}

\subsection{Modelo empírico}

Se parte de la base de la función de producción ampliada de Aschauer (1989):

$$
Y_{t}=A_{t} F\left(L_{t}, K_{t}, K G_{t}\right)
$$

Donde $Y_{t}$ es el valor añadido, $A_{t}$ es el índice de progreso técnico o productividad total de los factores, $L_{t}$ es el trabajo, $K_{t}$ el capital privado, y $K G_{t}$ el capital público.

En línea con Aschauer y otros autores posteriores, se supondrá que (1) es una función de producción del tipo Cobb-Douglas, por lo que podrá expresarse como ${ }^{6}$ :

$$
Y_{t}=A_{t} L_{t}^{\beta_{L}} K_{t}^{\beta_{K}} K G_{t}^{\beta_{G}}
$$

Dado que se pretende analizar el impacto del capital público sobre la producción privada a nivel regional y en presencia de capital humano y tecnológico se introducirán algunas modificaciones en (2). Concretamente, la función de producción ampliada tomará la siguiente forma:

$$
Y_{i t}=A_{i t} L_{i t}^{\beta_{L}} K_{i t-1}^{\beta_{K}} K G_{i t-1}^{\beta_{G}} K H_{i t-1}^{\beta_{H}} K T_{i t-1}^{\beta_{T}}
$$

Donde $K H$ es el capital humano y KT el capital tecnológico. Tomando logaritmos neperianos en (3) se obtiene una expresión fácilmente estimable:

$$
\ln Y_{i t}=\alpha_{i}+\beta_{L} \ln L_{i t}+\beta_{K} \ln K_{i t-1}+\beta_{G} \ln K G_{i t-1}+\beta_{H} \ln K H_{i t-1}+\beta_{T} \ln K T_{i t-1}+\varepsilon_{i t}
$$

Donde $\beta_{i}$ representa la elasticidad de la producción privada respecto al factor $i$-ésimo, $\varepsilon_{i t}$ es la perturbación aleatoria, y $\alpha_{i}$ hace referencia al término independiente de un modelo de datos de panel con efectos fijos para cada individuo (en este caso, cada comunidad autónoma). Como señalan Mas et al. (1996), puede interpretarse que los efectos fijos reflejan el conjunto particular de circunstancias que influyen en los resultados productivos de cada región y no son captados por los factores especificados en la función de producción, tales como las condiciones climáticas y estado del tiempo, la

\footnotetext{
6 Ver por ejemplo Munnell (1992), Gramlich (1994), De la Fuente y Vives (1995), Mas et al (1996), Sanaú (1997), Fernández y Polo (2002), Álvarez, Orea y Fernández (2003), Pérez Ximénez de Embún y Sanaú (2004), y otros.
} 
estructura productiva, el uso de los factores productivos, el uso de la tecnología, entre otros.

La ecuación (4) expresa la especificación genérica del modelo, es decir, la que incorpora el capital público total, $K G$, aunque se irá adaptando para incluir variantes tales como subdivisiones de éste en capital público económico y capital público social, y las diferentes categorías que conforman al primero de ellos. No se impondrán restricciones sobre el tipo de rendimientos a escala de la función de producción, sino que se contrastarán reparametrizando la ecuación (4) de la siguiente forma:

$$
\begin{aligned}
& \ln \left(\frac{Y_{i t}}{L_{i t}}\right)=\alpha_{i}+\left(\beta_{L}+\beta_{K}+\beta_{G}+\beta_{H}+\beta_{T}-1\right) \ln L_{i t}+\beta_{K} \ln \left(\frac{K_{i t-1}}{L_{i t}}\right)+ \\
& \beta_{G} \ln \left(\frac{K G_{i t-1}}{L_{i t}}\right)+\beta_{H} \ln \left(\frac{K H_{i t-1}}{L_{i t}}\right)+\beta_{T} \ln \left(\frac{K T_{i t-1}}{L_{i t}}\right)
\end{aligned}
$$

En la expresión (5), si el coeficiente que acompaña al empleo, $\left(\beta_{L}+\beta_{K}+\beta_{G}+\beta_{H}+\beta_{T}-1\right)$, goza de significatividad estadística y es mayor que cero, se interpretará como indicador de la presencia de rendimientos crecientes a escala en los factores; si es menor que cero se interpretará como indicador de rendimientos decrecientes; pero, si no es significativo, se interpretará como indicador de la presencia de rendimientos constantes a escala.

Asimismo, para contrastar la importancia de la composición del capital público se incluirá una nueva variante en la Ecuación (4), donde, en vez del stock de capital público total $\left(K G_{i t-1}\right)$ se introducirán dos categorías de éste, el stock de capital público económico $\left(K G E_{i t-1}\right)$, que incluye carreteras y autopistas, puertos, aeropuertos, ferrocarriles, infraestructuras hidráulicas y estructuras urbanas, y el stock de capital público social $\left(K G S_{i t-1}\right)$, que incluye infraestructuras para la educación y para la sanidad.

\subsection{Datos y fuentes}

Para la estimación del modelo empírico se formó un panel de datos con las variables $\mathrm{VAB}$, empleo, stock de capital físico privado, stock de capital público, capital humano y capital tecnológico, de las 17 comunidades autónomas españolas para el periodo 19882001. La definición y fuente de las variables es como sigue:

- VAB regional: (valor añadido bruto a precios de mercado) (expresado en miles de euros de 1990) ${ }^{7}$. Se tomaron los datos publicados por el Instituto Nacional de

${ }^{7}$ Todas las variables monetarias se expresaron en miles de euros de 1990 debido a que las cifras de capital público y privado publicadas por FBBVA-IVIE están dichas unidades y 
Estadística y se valoraron en euros de 1990, aplicando el deflactor del VAB y la paridad irrevocable entre la peseta y el euro.

- Empleo: Se tomaron los datos de la Contabilidad Regional de España que elabora el Instituto Nacional de Estadística.

- Stock de Capital Físico Privado: Se utilizó el stock de capital privado no residencial valorado en euros de 1990 calculado por el IVIE y publicado por la Fundación BBVA.

- Stock de Capital Público: Se usaron las series de la Fundación BBVA-IVIE expresadas en euros de 1990. Comprende nueve subcategorías: carreteras y autopistas, puertos, aeropuertos, ferrocarriles, infraestructuras hidráulicas, estructuras urbanas de corporaciones locales, educación, sanidad, y resto de funciones de las administraciones públicas. El capital público económico se calculó agregando carreteras y autopistas, infraestructuras hidráulicas, estructuras urbanas de corporaciones locales, puertos, ferrocarriles y aeropuertos. También se emplearon las series de capital social (educación y sanidad) y de capital público total (económico, social y resto de capital público). Estas series se extienden de 1964 a 2000, limitando el periodo de tiempo examinado en este trabajo.

- Capital Humano: Se aproximó con la población ocupada con estudios medios así como con la población ocupada con al menos estudios medios, obtenida al sumar la población con estudios medios, anteriores al superior, y superiores. Los datos están desagregados para cinco sectores de la economía y han sido calculados por el IVIE y publicados por Bancaja.

- Capital Tecnológico: Se emplearon tanto las cifras de personal en I+D en equivalencia a dedicación plena (EDP), como lo gastos internos en $\mathrm{I}+\mathrm{D}$, ambos del INE con los que se calcularon los stocks tecnológicos.

Los stocks se calcularon utilizando una función que acumula los gastos incurridos en la financiación de las actividades de I+D y descuenta una parte de los realizados en el pasado debido a la depreciación de los conocimientos y resultados tecnológicos. El método utilizado fue el del inventario permanente (MIP), que parte de un stock inicial, le añade anualmente el gasto en inversión bruta y le deduce la depreciación imputable.

Se supuso que los gastos en $\mathrm{I}+\mathrm{D}$ están sujetos a cierto retardo en su incorporación al stock, dado que los resultados de estas actividades no son inmediatos. Se partió de una función genérica, siguiendo la propuesta de Soete y Patel (1985), como $R_{t}=\sum \theta_{i} \cdot G I D_{t-1}$, donde $R_{t}$ es el stock de capital tecnológico en el período $t ; \theta_{i}$ se refiere a la estructura de retardos temporales con que se incorpora el gasto en $\mathrm{I}+\mathrm{D}$ al

debido a que los deflactores son aplicados sobre las series de inversión y no sobre los stocks estimados, luego es un ejercicio arduo el intentar cambiar el año base para el stock de capital. 
stock y también recoge la tasa de depreciación del capital tecnológico, y GID $_{t-1}$ es el gasto interno en $\mathrm{I}+\mathrm{D}$ en el periodo anterior a $t$.

Se dispuso de los datos de la "Estadística sobre las actividades de investigación científica y desarrollo tecnológico (I+D)" que elabora el INE. Estas series también se expresaron en unidades monetarias de 1990, al igual que las otras variables. En algunos trabajos se han propuesto deflactores propios del gasto en $\mathrm{I}+\mathrm{D}$, tarea excesivamente ardua en el caso de comunidades autónomas. Siguiendo otros trabajos se tomó el deflactor del PIB regional enlazando las series de la Contabilidad Regional base 1986 con las de la base 1995. Concretamente, se trabajó con las series de gasto interno en $\mathrm{I}+\mathrm{D}$ por comunidades autónomas y sectores (total, empresas, enseñanza superior, administración pública e instituciones privadas sin fines de lucro) para los años 1987 a 2001 en euros de $1990^{8}$.

Existen distintas formas de introducir los retardos en el modelo. Un método sencillo y habitualmente practicado en la literatura empírica es suponer que $\theta=2$, de manera que entre el gasto en I+D y su incorporación total al capital tecnológico transcurren dos años. Otros métodos incorporan progresivamente los gastos en $\mathrm{I}+\mathrm{D}$ al stock tecnológico.

En cuanto a la tasa de depreciación, se realizaron las estimaciones suponiendo tasas del $10 \%$ y $15 \%$. Para el trabajo se utilizó la del 10\%, aunque los resultados muestran escasa sensibilidad considerando una tasa de depreciación del 15\%.

Siguiendo el método propuesto por Griliches (1979) y utilizado por buena parte de la literatura empírica, el stock de capital inicial $R$ se calculó como:

$$
\left.\begin{array}{l}
\mathrm{R}_{\mathrm{i}, \mathrm{t+1}}=G I D_{l, t+1-\theta}+(1-\delta) \mathrm{R}_{\mathrm{i}, \mathrm{t}} \\
\mathrm{R}_{\mathrm{i}, \mathrm{t+1}}=\left(1+g_{i}\right) \cdot \mathrm{R}_{\mathrm{i}, \mathrm{t}}
\end{array}\right\} G I D_{l, t+1-\theta}+(1-\delta) \mathrm{R}_{\mathrm{i}, \mathrm{t}}=\left(1+g_{i}\right) \cdot \mathrm{R}_{\mathrm{i}, \mathrm{t}} \Rightarrow G I D_{l, t+1-\theta}=\left[1+g_{i}-(1-\delta)\right] \mathrm{R}_{\mathrm{i}, \mathrm{t}}
$$

de donde se obtiene $\mathrm{R}_{\mathrm{i}, \mathrm{t}}=\frac{G I D_{i, t+1-\theta}}{g_{i}+\delta}$, siendo $t$ el periodo inicial, $\mathrm{R}_{i, t}$ el stock inicial de capital tecnológico, $\theta$ la estructura de retardos, el retardo medio entre la realización de los gastos y la derivación de sus efectos (reduciendo de esta forma posibles sesgos de simultaneidad), $g_{i}$ la tasa media anual acumulativa de crecimiento de los gastos en $\mathrm{I}+\mathrm{D}^{9}$ y pagos por patentes de la región $i$ durante un período y $\delta$ la tasa de depreciación del stock tecnológico (10 ó 15\%). En este caso la fórmula para el cálculo del stock inicial,

\footnotetext{
${ }^{8}$ En el concepto gastos internos se incluyen tanto los gastos de capital como los gastos corrientes.

9 Sería más relevante la inclusión también de los pagos por patentes tecnológicas pero no se dispone de estos datos regionalizados.
} 
que correspondería al año 1988, para la región $i$ sería la siguiente $\mathrm{R}_{\mathrm{i}, 88}=\frac{G I D_{i, t+1-2}}{g_{i}+\delta}=\frac{G I D_{i, 87}}{g_{i}+\delta}$.

Como paso previo a la estimación del modelo, a continuación se ofrece un análisis preliminar de los datos de las variables más relevantes empleadas en este trabajo, para lo que se presenta un conjunto de estadísticos descriptivos de las variables más relevantes en el Cuadro 1. El Cuadro 2, por su parte, presenta un primer análisis descriptivo de las dotaciones regionales de capital público.

\section{CUADRO 1 \\ ESTADÍSTICOS DESCRIPTIVOS DE VARIABLES MÁS RELEVANTES.}

\begin{tabular}{|l|c|c|c|c|c|}
\hline & Media & Mediana & $\begin{array}{c}\text { Desviación } \\
\text { Estándar }\end{array}$ & Mínimo & Máximo \\
\hline VAB & $18.349 .716,7$ & $10.922 .330,0$ & $17.035 .027,4$ & $1.968 .041,4$ & $70.408 .238,2$ \\
L & 812,2 & 495,4 & 702,6 & 97,3 & $2.827,6$ \\
K & $43.416 .900,6$ & $26.032 .656,7$ & $39.952 .766,0$ & $4.006 .399,0$ & $169.628 .209,1$ \\
KG & $9.475 .427,6$ & $7.100 .873,5$ & $7.335 .508,3$ & $1.551 .371,2$ & $33.250 .177,3$ \\
KGE & $6.081 .885,1$ & $4.591 .835,0$ & $4.746 .255,9$ & $897.932,3$ & $21.537 .327,0$ \\
Carretaut & $2.735 .292,6$ & $2.147 .179,7$ & $2.090 .703,1$ & $288.984,9$ & $8.865 .112,6$ \\
KH & 347,9 & 209,4 & 326,4 & 24,6 & $1.601,9$ \\
KT & $1.035 .176,6$ & $304.283,9$ & $1.875 .901,3$ & $3.510,8$ & $9.047 .499,5$ \\
\hline Nota: $V A B$ es el valor añadido bruto, $L$ el empleo, K el stock de capital físico privado, KG el stock \\
de capital público total, KGE el stock de capital público económico, Carretaut el stock de capital \\
público en carreteras y autopistas, KH el capital humano y KT el capital tecnológico. \\
\hline \multicolumn{7}{|l}{} \\
\hline
\end{tabular}

En el Cuadro 2 se incluyen variables como la media en la dotación de capital público para el periodo 1988-2001, la posición relativa de cada región, la tasa media de variación anual acumulativa (TMVA), la media de la dotación normalizada por el producto de la población y la superficie de la región (Med (P, S), y un nuevo ranking para este indicador. Se cree que los dos últimos están mucho más cerca de la realidad económica y más relacionados con el concepto de productividad. Las cinco variables se muestran en función del capital público total y también del capital público económico.

Pueden destacarse varios aspectos interesantes del análisis recogido en el Cuadro 2. En primer lugar, las comunidades autónomas con mayor dotación de capital público total para el periodo analizado son, por orden, Andalucía, Cataluña, Madrid, la Comunidad Valenciana, y Castilla y León. En cuanto al capital público económico, las cinco 
regiones más dotadas siguen siendo las mismas, salvo una alteración en el orden, en el que Madrid y la Comunidad Valenciana alternan sus posiciones relativas.

Sin embargo, si se observa la columna de la media normalizada por la población y la superficie para el capital público total, las posiciones se modifican sensiblemente hasta el punto de que tres de las mejor situadas en la clasificación anterior (Andalucía, Cataluña, y Castilla y León), pasan a ser de las peor dotadas. Asimismo, las cuatro regiones que ocupan los últimos lugares según la primera clasificación, como son La Rioja, Baleares, Cantabria, y Navarra, pasan a ser unas de las mejor dotadas en cuanto a la segunda clasificación.

\section{CUADRO 2}

\section{DOTACIONES REGIONALES DE CAPITAL PÚBLICO 1988-2001}

\begin{tabular}{|c|c|c|c|c|c|c|c|c|c|c|}
\hline \multirow[b]{2}{*}{ CC.AA. } & \multicolumn{5}{|c|}{ CAPITAL PÚBLICO } & \multicolumn{5}{|c|}{ CAPITAL PÚBLICO ECONÓMICO } \\
\hline & Media & $\begin{array}{c}\text { Ran } \\
\mathrm{k}\end{array}$ & $\begin{array}{c}\text { TMV } \\
\text { A }\end{array}$ & $\begin{array}{l}\text { Med } \\
(\mathrm{P}, \mathrm{S})\end{array}$ & $\begin{array}{c}\text { Ran } \\
\mathrm{k}\end{array}$ & Media & $\begin{array}{c}\text { Ran } \\
k\end{array}$ & $\begin{array}{c}\text { TMV } \\
\text { A }\end{array}$ & $\begin{array}{l}\text { Med } \\
(\mathrm{P}, \mathrm{S})\end{array}$ & $\begin{array}{c}\text { Ran } \\
\mathrm{k}\end{array}$ \\
\hline Andalucía & 26.425 .8 & 1 & 5,58 & 0,041 & 17 & 16.739 .6 & 1 & 5,89 & 0,026 & 17 \\
\hline Aragón & 55 & 9 & $\%$ & 3 & 12 & 37 & 9 & $\%$ & 2 & 11 \\
\hline Asturias & 6.991 .17 & 11 & 3,21 & 0,123 & 7 & 4.928 .58 & 10 & 2,81 & 0,086 & 6 \\
\hline Baleares & 4 & 16 & $\%$ & 2 & 5 & 3 & 17 & $\%$ & 9 & 5 \\
\hline Canarias & 5.265 .47 & 10 & 5,01 & 0,459 & 6 & 3.535 .63 & 11 & 4,48 & 0,308 & 7 \\
\hline Cantabria & 0 & 15 & $\%$ & 3 & 2 & 7 & 16 & $\%$ & 4 & 2 \\
\hline Castilla y & 2.392 .06 & 5 & 5,56 & 0,583 & 16 & 1.365 .23 & 5 & 4,96 & 0,333 & 16 \\
\hline León & 2 & 8 & $\%$ & 9 & 15 & 1 & 8 & $\%$ & 3 & 15 \\
\hline Castilla-La & 6.185 .98 & 2 & 5,84 & 0,494 & 14 & 3.457 .60 & 2 & 5,02 & 0,276 & 13 \\
\hline Mancha & 2 & 4 & $\%$ & 0 & 10 & 1 & 3 & $\%$ & 1 & 10 \\
\hline Cataluña & 2.430 .68 & 12 & 5,91 & 0,872 & 13 & 1.538 .26 & 12 & 6,71 & 0,552 & 14 \\
\hline Com. & 7 & 6 & $\%$ & 5 & 11 & 5 & 7 & $\%$ & 1 & 12 \\
\hline Valenciana & 13.079 .1 & 3 & 3,82 & 0,056 & 8 & 8.634 .26 & 4 & 3,53 & 0,037 & 8 \\
\hline Extremadu & 34 & 13 & $\%$ & 0 & 9 & 3 & 13 & $\%$ & 0 & 9 \\
\hline $\mathrm{ra}$ & 8.496 .23 & 14 & 4,91 & 0,061 & 4 & 5.915 .32 & 14 & 5,10 & 0,043 & 3 \\
\hline Galicia & 3 & 7 & $\%$ & 9 & 3 & 9 & 6 & $\%$ & 1 & 4 \\
\hline Madrid & 22.558 .3 & 17 & 4,60 & 0,113 & 1 & 15.457 .8 & 15 & 4,16 & 0,077 & 1 \\
\hline Murcia & 92 & & $\%$ & 1 & & 27 & & $\%$ & 5 & \\
\hline Navarra & 15.060 .2 & & 4,91 & 0,158 & & 10.169 .8 & & 4,30 & 0,107 & \\
\hline País Vasco & 73 & & $\%$ & 6 & & 92 & & $\%$ & 1 & \\
\hline Rioja, La & 5.078 .55 & & 5,61 & 0,113 & & 3.026 .67 & & 4,68 & 0,067 & \\
\hline & 3 & & $\%$ & 9 & & 7 & & $\%$ & 9 & \\
\hline & 11.027 .5 & & 5,86 & 0,136 & & 6.493 .61 & & 5,63 & 0,080 & \\
\hline & 77 & & $\%$ & 5 & & 1 & & $\%$ & 4 & \\
\hline
\end{tabular}




\begin{tabular}{|c|c|c|c|c|c|c|c|}
\hline & $\begin{array}{c}15.887 .8 \\
55 \\
3.740 .15 \\
5 \\
3.489 .59 \\
1 \\
10.945 .4 \\
90 \\
2.027 .78 \\
7\end{array}$ & $\begin{array}{c}4,87 \\
\% \\
5,70 \\
\% \\
3,52 \\
\% \\
3,94 \\
\% \\
0,42 \\
\%\end{array}$ & $\begin{array}{c}0,383 \\
3 \\
0,290 \\
9 \\
0,661 \\
9 \\
0,735 \\
4 \\
1,518 \\
1\end{array}$ & $\begin{array}{c}9.070 .12 \\
4 \\
2.469 .28 \\
0 \\
2.380 .65 \\
9 \\
6.657 .45 \\
1 \\
1.551 .97 \\
9\end{array}$ & $\begin{array}{c}4,43 \\
\% \\
5,59 \\
\% \\
2,54 \\
\% \\
2,67 \\
\% \\
- \\
0,80 \\
\%\end{array}$ & $\begin{array}{c}0,218 \\
8 \\
0,192 \\
0 \\
0,451 \\
6 \\
0,447 \\
3 \\
1,161 \\
9\end{array}$ & \\
\hline $\begin{array}{l}\text { Notas: Media } \\
\text { 1990; TMVA } \\
\text { normalizada p }\end{array}$ & $\begin{array}{l}\text { es la dotaci } \\
\text { es la tasa } \\
\text { r el produc }\end{array}$ & blaci & a st & a región c & ente. & $\begin{array}{l}\text { es de e } \\
\text { ión pr }\end{array}$ & Iros de \\
\hline
\end{tabular}

A la luz de lo anterior, podría concluirse que las comunidades con mayores dotaciones de capital público (quizá con la excepción de Madrid) tienen más población y/o extensión. El caso más interesante resulta ser el de Andalucía que pasa del primero al último puesto al comparar los dos indicadores. La tasa media de variación anual acumulativa ilustra como las comunidades en donde más ha crecido anualmente el stock de capital público total son Cantabria, Galicia, Canarias, Murcia, Extremadura y Andalucía, en contraste con regiones como La Rioja, Aragón, Navarra, Castilla y León, y País Vasco en donde han sido menores.

Este análisis resulta igualmente válido para el caso del capital público económico, salvo algunas pequeñas alternancias en las posiciones relativas de algunas comunidades, por ejemplo, entre Cataluña y Extremadura, y entre Aragón y Galicia. El comportamiento de la tasa de variación anual acumulativa también guarda estrecha relación con el del capital público total, lo que no permite concluir, en términos generales, que las regiones con menores dotaciones en los dos tipos de capital público sean las que han experimentado las mayores tasas de crecimiento en sus stocks correspondientes, y viceversa. A continuación se indagará acerca de las posibles regularidades estadísticas existentes entre el capital público y la productividad privada, en presencia de capital humano y tecnológico.

\section{ESTIMACIÓN Y RESULTADOS}

Con el fin de dar cumplimiento a los objetivos planteados en este trabajo se ha construido un modelo econométrico con un panel de datos de las regiones españolas para el periodo 1988-2001, en el que se puedan especificar las dimensiones espacial y 
temporal que requiere el análisis, con un modelo donde el output de la región $i$ en el año $t$ no sólo dependa de las cantidades de trabajo y capital privado y público usados, sino también de los capitales humano y tecnológico. Esto último en razón de que los recientes desarrollos tanto teóricos como empíricos de la literatura sobre crecimiento económico sugieren que estos dos stocks están asociados con incrementos del output y de la productividad. Este modelo, que se refleja en la Ecuación (4), se ha estimado por MCO controlando para auto correlación de orden $1 .{ }^{10}$

Pese a que resulta usual en la literatura sobre esta materia el uso de modelos de efectos fijos, se estimaron los modelos de efectos fijos y el restringido y se contrastaron estadísticamente. Para tal fin se utilizó el Contraste de Significatividad de los Efectos de Grupo empleado por Greene (1999), rechazándose en todos los casos la hipótesis de que los efectos de las comunidades autónomas son iguales a un nivel de significatividad del 1\%, evidencia a favor de la utilización del modelo de efectos fijos. Los resultados de dichos contrastes se presentan en los Cuadros 4 y $6 .{ }^{11}$

\subsection{Estimaciones con datos agregados de las regiones españolas}

A partir de la estimación del modelo empírico planteado se pretende aportar evidencia empírica, ya sea a favor o en contra sobre la hipótesis del capital público a través de tres objetivos puntuales: 1) estimar el efecto del capital público total sobre la producción agregada a nivel regional en presencia de capital humano y tecnológico, sin imponer restricciones sobre el tipo de rendimientos presente en la función de producción; 2) analizar la importancia de la composición del capital público (económico y social), y 3) evaluar si existen spillovers en este tipo de inversión, es decir, si se presenta el "efecto desbordamiento" en el capital público y algunas subcategorías de éste ${ }^{12}$.

\footnotetext{
10 Siguiendo a Pérez y Sanaú (2004), entre otros, se introdujeron las variables “capital” retardadas un periodo.

${ }^{11}$ El estadístico de contraste utilizado fue: $F(n-1, n T-n-K)=\frac{\left[\left(R_{f}^{2}-R_{c}^{2}\right) /(n-1)\right]}{\left[\left(1-R_{f}^{2}\right) /(n T-n-K)\right]}$

12 La estimación econométrica de este modelo se realizó mediante el programa Eviews 5.0.
} 


\section{CUADRO 3 \\ CAPITAL PÚBLICO Y PRODUCCIÓN}

\begin{tabular}{|c|c|}
\hline Variables & 1 \\
\hline \multirow[t]{2}{*}{$\mathrm{L}$} & 0,046 \\
\hline & $(0,668)$ \\
\hline \multirow[t]{2}{*}{$\mathrm{K} / \mathrm{L}$} & 0,390 \\
\hline & $(3,747)^{* *}$ \\
\hline \multirow[t]{2}{*}{$\mathrm{KG} / \mathrm{L}$} & 0,156 \\
\hline & $(2,703) * *$ \\
\hline \multirow[t]{2}{*}{$\mathrm{KH} / \mathrm{L}$} & 0,117 \\
\hline & $(2,806)^{* *}$ \\
\hline $\mathrm{KT} / \mathrm{L}$ & $\begin{array}{c}-0,058 \\
(-2320) * *\end{array}$ \\
\hline $\mathrm{R}^{2}$ & 0,975 \\
\hline $\mathrm{F}$ & 333,1 \\
\hline D-W & 2,13 \\
\hline \multicolumn{2}{|c|}{$\begin{array}{l}\text { Notas: 1) Los valores entre } \\
\text { paréntesis son estadísticos t. } * \\
\text { Significatividad al } 10 \%, * * \\
\text { Significatividad al } 5 \% .2) L \\
\text { representa el empleo, } K \text { el stock de } \\
\text { capital físico privado, } K G \text { el stock de } \\
\text { capital público total, } K H \text { el capital } \\
\text { humano y } K T \text { el capital tecnológico. }\end{array}$} \\
\hline
\end{tabular}

En correspondencia con el primero de los objetivos mencionados arriba, se contrastó el tipo de rendimientos de la función de producción. En la estimación de la expresión (5) la ausencia de significatividad del coeficiente que acompaña al empleo $\left(\beta_{L}+\beta_{K}+\beta_{G}+\beta_{H}+\beta_{T}-1\right)$ puede interpretarse como indicio de la presencia de rendimientos constantes a escala en todos los factores. Los resultados de la estimación se muestran en el Cuadro 3, e indican que la función de producción presenta rendimientos constantes a escala (RCE). El parámetro que acompaña al factor trabajo $(0,046)$ no es estadísticamente significativo a los niveles usuales, lo que no permite rechazar la hipótesis nula de existencia de rendimientos constantes en todos los factores $\left(\beta_{L}+\beta_{K}+\beta_{G}+\beta_{H}+\beta_{T}=1,046\right)$. Por lo tanto, en lo que sigue se impondrán RCE.

\subsubsection{Composición del capital público}

Suele admitirse que ciertas categorías del capital público están más estrechamente relacionadas con los procesos productivos que otras; de ahí el interés por contrastar si 
el efecto del capital público sobre la productividad es diferente dependiendo de su composición.

En el Cuadro 4 se presentan los resultados de la estimación de las ecuaciones (4) y (6) suponiendo rendimientos constantes a escala. Los resultados de estas primeras dos columnas permiten rechazar la hipótesis de Fernández y Polo (2001 y 2002), pues la inclusión del capital humano y el capital tecnológico en el modelo estimado apenas altera el parámetro de $K G$ y de $K G E$ (no los reduce, antes los aumenta). Para el caso del capital público económico (KGE) la elasticidad es 0,133 cuando no se incluyen los capitales humano y tecnológico, y 0,138 cuando se incluyen (ambos estadísticamente significativos).

\section{CUADRO 4}

COMPOSICIÓN DEL CAPITAL PÚBLICO. Modelo de efectos fijos

\begin{tabular}{|c|c|c|c|c|c|c|}
\hline Variables & 1 & 2 & 3 & 4 & 5 & 6 \\
\hline \multirow[t]{2}{*}{$\mathrm{L}$} & 0,495 & 0,492 & 0,441 & 0,431 & 0,438 & 0,442 \\
\hline & $(9,493) * *$ & $(9,433) * *$ & $(8,045)^{* *}$ & $(7,831)^{* *}$ & $(7,991)^{* *}$ & $(7,871)^{* *}$ \\
\hline \multirow[t]{2}{*}{ K } & 0,401 & 0,422 & 0,390 & 0,439 & 0,405 & 0,482 \\
\hline & $(4,361)^{* *}$ & $(4,886) * *$ & $(3,747)^{* *}$ & $(4,153)^{* *}$ & $(3,942) * *$ & $(4,455)^{* *}$ \\
\hline KG & $\begin{array}{c}0,146 \\
(2,510) * *\end{array}$ & & $\begin{array}{c}0,156 \\
(2,703) * *\end{array}$ & & & \\
\hline KGE & & $\begin{array}{c}0,133 \\
(2,531)^{* *}\end{array}$ & & $\begin{array}{c}0,175 \\
(2,940) * *\end{array}$ & $\begin{array}{c}0,138 \\
(2,649) * *\end{array}$ & \\
\hline KGS & & & & $\begin{array}{c}-0,101 \\
(-1,264)\end{array}$ & & $\begin{array}{c}0,023 \\
(0,327)\end{array}$ \\
\hline \multirow[t]{2}{*}{$\mathrm{KH}$} & & & 0,117 & 0,125 & 0,118 & 0,124 \\
\hline & & & $(2,806)^{* *}$ & $(2,963)^{* *}$ & $(2,822)^{* *}$ & $(2,861)^{* *}$ \\
\hline \multirow[t]{2}{*}{$\mathrm{KT}$} & & & $-0,058$ & $-0,039$ & $-0,054$ & $-0,042$ \\
\hline & & & $(-2,320) * *$ & $(-1,418)$ & $(-2,148)^{* *}$ & $(-1,448)$ \\
\hline $\mathrm{R}^{2}$ & 0,999 & 0,999 & 0,999 & 0,999 & 0,999 & 0,999 \\
\hline $\mathrm{F}$ & 18771,8 & 18786,7 & 17864,4 & 17146,4 & 17837,9 & 17185,5 \\
\hline D-W & 2,03 & 2,03 & 2,13 & 2,14 & 2,12 & 2,08 \\
\hline \multicolumn{7}{|c|}{$\begin{array}{l}\text { Notas: 1) Los valores entre paréntesis son estadísticos } t \text {. * Significatividad al } 10 \% \text {, ** } \\
\text { Significatividad al } 5 \% \text {. 2) } L \text { representa el empleo, } K \text { el stock de capital físico privado, } K G \text { el stock } \\
\text { de capital público total, KGE el stock de capital público económico, KGS el stock de capital } \\
\text { público social, } K H \text { el capital humano y } K T \text { el capital tecnológico. 3) A partir del Contraste de } \\
\text { Significatividad de los Efectos de Grupo se obtuvieron los siguientes resultados: para el modelo } \\
\text { con capital público total }(K G) \text { una } F \text { de } 2,79 \text {; para el modelo de composición del capital público } \\
(K G E \text { y } K G S) \text { una } F \text { de } 2,67 \text {; para el de capital público económico }(K G E) \text { una } F \text { de } 2,73 \text {. El valor } \\
\text { crítico al } 1 \% \text { de la tabla } F \text { es } 2,04 \text {. }\end{array}$} \\
\hline
\end{tabular}


En la columna 3 del mencionado Cuadro se muestran los resultados con la ecuación general (4) donde aparece una elasticidad positiva y estadísticamente significativa para el capital público total de 0,156 , la cual resulta mucho menor que la del capital físico privado, en línea con la mayoría de trabajos anteriores. Ha de resaltarse el comportamiento de las "nuevas variables". El coeficiente del capital humano exhibe un razonable valor positivo y significativo aunque menor que el del capital público y que el del capital físico privado. En cambio, la elasticidad del producto con respecto al capital tecnológico presenta una magnitud negativa y estadísticamente significativa.

En la columna 4 se muestran los resultados de la estimación al desagregar el capital público en sus dos grandes categorías. Obsérvese que hay claras diferencias en los efectos del capital público económico y el social, como habían concluido otros autores antes. Mas et al. (1996), por ejemplo, hallaron diferencias reveladoras entre lo que llamaron capital público productivo y capital público social, obteniendo valores para los parámetros de 0,086 y $-0,021$ respectivamente, si bien este último no era estadísticamente significativo.

El valor de nuestro parámetro resulta notoriamente mayor que el obtenido por Mas et al. (1996). La elasticidad de la producción con respecto a KGE de la columna 4 se sitúa en 0,175 mientras que aquella con respecto a $K G S$ resulta negativa aunque no estadísticamente significativa a los niveles usuales. Al estimar (6) tomando por separado a los dos tipos de infraestructuras (columnas 5 y 6 ) las diferencias son evidentes y confirman la diferencia en los efectos del capital público económico frente al social, demostrando que el primero es el que se relaciona directamente con el VAB.

\subsubsection{Subcategorías del capital público}

Puesto que no es habitual que se presenten resultados que relacionen la producción del sector privado con subcategorías específicas del capital público, en este apartado se usan los datos del capital público desagregados en seis subcategorías de naturaleza económica y dos de naturaleza social para observar el impacto de cada una de ellos separadamente. El Cuadro 5 incluye los resultados de ocho diferentes especificaciones en las que los factores trabajo, capital privado, capital humano y capital tecnológico son los habituales, y como capital público se consideran individualmente las distintas subcategorías. Lo primero a destacar es la relativa estabilidad de los parámetros que acompañan a todos los demás factores (trabajo, capital privado, capital humano y capital tecnológico) a excepción de las columnas 3 y 4 que corresponden a aeropuertos y ferrocarriles respectivamente. 


\section{CUADRO 5}

\section{SUBCATEGORÍAS DEL CAPITAL PÚBLICO}

\begin{tabular}{|c|c|c|c|c|c|c|c|c|c|c|}
\hline Variables & 1 & 2 & 3 & 4 & 5 & 6 & 7 & 8 & 9 & 10 \\
\hline$L$ & 0,451 & 0,470 & 0,553 & 0,441 & 0,459 & 0,437 & 0,449 & 0,443 & 0,446 & 0,442 \\
\hline & $(8,018)^{\star *}$ & $(7,849)^{\star *}$ & $(11,474)^{\star *}$ & $(7,895)^{\star *}$ & $(8,506)^{\star *}$ & $(7,910)^{\star \star}$ & $(7,955)^{\star *}$ & $(7,932)^{\star *}$ & $(8,101)^{\star *}$ & $(8,075)^{\star *}$ \\
\hline$K$ & 0,471 & 0,406 & 0,450 & 0,509 & 0,472 & 0,412 & 0,439 & 0,506 & 0,470 & 0,429 \\
\hline Carretaut & $\begin{array}{c}(4,736)^{\star \star x} \\
\mathbf{0 , 0 6 5}\end{array}$ & $(3,196)^{\star \star}$ & $(4,505)^{\star \star}$ & $(4,893)^{\star *}$ & $(4,971)^{\star \star}$ & $(3,673)^{\star *}$ & $(4,029)^{\star *}$ & $(5,000)^{\star *}$ & $(4,844)^{\star *}$ & $(4,335)^{\star *}$ \\
\hline carrelaut & $(1,775)^{\star}$ & & & & & & & & & \\
\hline Ptos & & $\begin{array}{c}0,018 \\
(0,193)\end{array}$ & & & & & & & & \\
\hline Arptos & & & $\begin{array}{c}0,024 \\
(2,321)^{\star *}\end{array}$ & & & & & & & \\
\hline Ferroc & & & & $\begin{array}{c}-0,011 \\
(-0,500)\end{array}$ & & & & & & \\
\hline InfHid & & & & & $\begin{array}{c}0,132 \\
(3,524)^{\star \star}\end{array}$ & & & & & \\
\hline EstUrb & & & & & & 0,040 & & & & \\
\hline Edur & & & & & & $(1,554)$ & & & & \\
\hline Eauc & & & & & & & $\begin{array}{c}0,081 \\
(1,252)\end{array}$ & & & \\
\hline Sanid & & & & & & & & $-0,036$ & & \\
\hline $\begin{array}{l}\text { Carretaut+Arptos+ } \\
\text { InfHid }\end{array}$ & & & & & & & & $(-0,897)$ & $\begin{array}{c}0,117 \\
(2,633)^{\star \star}\end{array}$ & \\
\hline $\begin{array}{l}\text { Carretaut+Arptos+ } \\
\text { InfHid+EstUrb }\end{array}$ & & & & & & & & & & $\begin{array}{c}0,129 \\
(2,791)^{\star \star}\end{array}$ \\
\hline$K H$ & $\begin{array}{c}0,123 \\
(2,906)^{* *}\end{array}$ & $\begin{array}{c}0,051 \\
(1,208)\end{array}$ & $\begin{array}{c}0,032 \\
(0,905)\end{array}$ & $\begin{array}{c}0,125 \\
(2,922)^{* *}\end{array}$ & $\begin{array}{c}0,111 \\
(2,695)^{\star *}\end{array}$ & $\begin{array}{c}0,132 \\
(3,116)^{\star \star}\end{array}$ & $\begin{array}{c}0,119 \\
(2,780)^{\star *}\end{array}$ & $\begin{array}{c}0,127 \\
(2,966)^{* *}\end{array}$ & $\begin{array}{c}0,114 \\
(2,713)^{\star \star}\end{array}$ & $\begin{array}{c}0,116 \\
(2,789)^{\star *}\end{array}$ \\
\hline$K T$ & $\begin{array}{c}-0,051 \\
(-1.942)^{*}\end{array}$ & $\begin{array}{c}0,035 \\
(0,848)\end{array}$ & $\begin{array}{l}-0,015 \\
(-0,509)\end{array}$ & $\begin{array}{l}-0,040 \\
(-1,501)\end{array}$ & $\begin{array}{c}-0,063 \\
-2524)^{\star *}\end{array}$ & $\begin{array}{c}-0,044 \\
(-1753)^{*}\end{array}$ & $\begin{array}{c}-0,054 \\
(-1,909)^{*}\end{array}$ & $\begin{array}{l}-0,030 \\
(-1,114)\end{array}$ & $\begin{array}{l}-0,060 \\
-2,339)^{\star *}\end{array}$ & $\begin{array}{c}-0,060 \\
-2,376)^{\star *}\end{array}$ \\
\hline $\mathrm{R}^{2}$ & 0,999 & 0,999 & 0,999 & 0,999 & 0,999 & 0,999 & 0,999 & 0,999 & 0,999 & 0,999 \\
\hline $\mathrm{F}$ & 17469,9 & 22282,0 & 21467,6 & 17198,0 & 18290,9 & 17379,8 & 17315,0 & 17246,3 & 17844,7 & 17924,5 \\
\hline D-W & 2,12 & 2,17 & 2,08 & 2,09 & 2,16 & 2,10 & 2,07 & 2,09 & 2,14 & 2,14 \\
\hline
\end{tabular}


Sin embargo, se observan notables diferencias en el valor del parámetro que acompaña al capital público, así como en el t-estadístico. Las categorías más relevantes parecen ser infraestructuras hidráulicas, infraestructuras para la educación, carreteras y autopistas, estructuras urbanas, aeropuertos, y puertos; sin embargo, de estas seis, solamente las infraestructuras hidráulicas, carreteras y autopistas, y aeropuertos logran significatividad estadística.

Es preciso resaltar, que aunque las estructuras urbanas no alcanzan significatividad, si se añade esta categoría a las carreteras y autopistas, los aeropuertos y las infraestructuras hidráulicas y se estiman las cuatro como una sola ocurren dos cosas importantes: la elasticidad de la productividad del sector privado con respecto a este nuevo género de capital público pasa de $11,7 \%$ a $12,9 \%$, y el valor del estadístico $t$ también aumenta. La columna 9 del Cuadro 5 muestra los resultados de la estimación en el que la variable $K G_{\text {rit-1 }}$ agrupa a los tres tipos de capital público que dieron significativos en las columnas 1-8; en la columna 10 se añaden las estructuras urbanas a estas tres y se muestran los resultados ya comentados.

Lo anterior, sumado al hecho de que algunos tipos de infraestructuras (cuando se examinan separadamente) tienen un efecto menor, o en algunos casos intrascendente, sobre el nivel de producción en contraste con el que muestran cuando se tienen en cuenta conjuntamente puede deberse al "efecto cooperación o sinérgico", el cual sugiere que los efectos en la actividad económica de un conjunto de infraestructuras suelen ser mayores que los de las diferentes subcategorías individualmente consideradas, tal como indica Sanaú (1995).

\subsubsection{Efecto desbordamiento del capital público}

Teniendo en cuenta que la producción de una región, por ejemplo Aragón, puede verse influida no solamente por el stock de capital público localizado en esa comunidad autónoma sino también por la red total del territorio español, y especialmente por las dotaciones de las regiones más próximas a ella, trabajos como el de Mas et al. (1996) estudiaron la existencia de spillovers en el capital público. Para probar el "efecto desbordamiento" con datos más elaborados y un periodo temporal más reciente, aquí se empleó una nueva especificación para tres tipos distintos de capital público (total, económico, y carreteras y autopistas), diferente a la de Mas et al. (1996), porque consideramos que no sólo es importante el capital público de las regiones adyacentes sino que también puede influir el de otras regiones menos próximas. La tipificación propuesta es una modificación de la que ha sido utilizada por Álvarez, Orea y Fernández (2003), que ponderaban el capital público por la distancia que separa las 
capitales de las distintas comunidades autónomas ${ }^{13}$. En nuestro caso, la variable ampliada de capital público para la región $i$ será:

$$
K G d_{i s}=K G_{i s}+\sum_{1}^{N-1} e^{\left(-D_{i m}\right)} * K G_{m s}
$$

Donde $D_{i m}$ es la distancia entre la región $m$ y la región analizada $i, s=1,2,3$ (el número de categorías del capital público evaluadas); y $N$ es el número total de regiones. Se supone que el efecto desbordamiento del capital público decrece con la distancia, es decir que si se analiza a la comunidad de Aragón, la medida expresada aquí dará más peso a las dotaciones de las comunidades Valenciana, Catalana, y Navarra que a las de Andalucía o Galicia, por ejemplo.

\section{CUADRO 6 \\ EFECTO DESBORDAMIENTO DEL CAPITAL PÚBLICO}

\begin{tabular}{|c|c|c|c|}
\hline Variables & 1 & 2 & 3 \\
\hline $\mathrm{L}$ & 0,439 & 0,432 & 0,445 \\
\hline & $(7,962)^{* *}$ & $(7,902)^{* *}$ & $(7,966) * *$ \\
\hline K & 0,370 & 0,376 & 0,425 \\
\hline & $(3,302)^{* *}$ & $(3,488)^{* *}$ & $(4,173)^{* *}$ \\
\hline KGd & $\begin{array}{c}0,177 \\
(2,166) * *\end{array}$ & & \\
\hline KGEd & & $\begin{array}{c}0,186 \\
(2,341)^{* *}\end{array}$ & \\
\hline Carretautd & & & $\begin{array}{c}0,125 \\
(2,037)^{* *}\end{array}$ \\
\hline $\mathrm{KH}$ & $\begin{array}{c}0,133 \\
(3,190)^{* *}\end{array}$ & $\begin{array}{c}0,132 \\
(3,173)^{* *}\end{array}$ & $\begin{array}{c}0,141 \\
(3,368)^{* *}\end{array}$ \\
\hline $\mathrm{KT}$ & $\begin{array}{c}-0,074 \\
(-2,655)^{* *}\end{array}$ & $\begin{array}{c}-0,076 \\
(-2,757)^{* *}\end{array}$ & $\begin{array}{c}-0,068 \\
(-2,511)^{* *}\end{array}$ \\
\hline$\overline{\mathrm{R}^{2}}$ & 0,999 & 0,999 & 0,999 \\
\hline F & 17546,1 & 17601,2 & 17506,4 \\
\hline $\mathrm{D}-\mathrm{W}$ & 2,10 & 2,09 & 2,09 \\
\hline \multicolumn{4}{|c|}{$\begin{array}{l}\text { Notas: 1) Los valores entre paréntesis son estadísticos t. } \\
* \text { Significatividad al } 10 \% \text {; ** Significatividad al 5\%. 2) KGd representa el stock de capital } \\
\text { público total ampliado, KGEd el stock de capital público económico ampliado, Carretautd el } \\
\text { stock de capital público ampliado en carreteras y autopistas, y las demás variables según se } \\
\text { han descrito antes. 3) A partir del Contraste de Significatividad de los Efectos de Grupo se } \\
\text { obtuvieron los siguientes resultados: para el modelo de los "efectos desbordamiento" de } \\
\text { KG una F de 2,49; y para el de "efectos desbordamiento" de KGE una F de 2,56. El valor } \\
\text { crítico al } 1 \% \text { de la tabla F es 2,04. }\end{array}$} \\
\hline
\end{tabular}

${ }^{13}$ En contraste, en este trabajo se ha considerado incluir la principal ciudad de cada comunidad autónoma (en función del VAB generado en cada una de ellas) dado que no siempre la capital de una comunidad autónoma es la ciudad más importante en términos de producción agregada. 
El Cuadro 6 muestra los resultados de la estimación de (4), reemplazando la variable capital público por $K G d_{\text {is }}$ para evaluar el efecto desbordamiento en el capital público total, el económico, y las carreteras y autopistas. Los resultados parecen concluyentes, ya que se observa que el valor de la elasticidad para el capital público total ampliado es 0,177, mayor que el obtenido cuando sólo es considerada la dotación de cada región, 0,156. Asimismo, para el caso del capital público económico (las dotaciones más relacionadas con los procesos productivos) el valor de la elasticidad aumenta, pero esta vez en mayor proporción que el anterior, pues pasa de 0,138 a 0,186. Sin embargo, y como era de esperar, el mayor aumento se presentó en el caso de las carreteras y autopistas, pasando la elasticidad del output con relación a esta categoría de 0,065 a 0,125 .

A partir de los resultados obtenidos, en línea con Mas et al. (1996) y Álvarez et al. (2003), puede concluirse que el aumento en la elasticidad asociada al capital público cuando se tiene en cuenta la variable ampliada con las dotaciones de las demás regiones del país ponderadas por la distancia, parece confirmar la hipótesis del efecto desbordamiento.

\subsection{Estimaciones con datos sectoriales de las regiones españolas}

Como se señaló, en este trabajo se pretende profundizar en el conocimiento del capital público económico y su relación con la productividad de los sectores más importantes de la economía, asunto que no se ha abordado con la suficiente profundidad en la literatura previa. En este contexto, el modelo empírico se estimó con datos desagregados de cinco sectores de la economía española: agricultura, energía, industria, construcción, y servicios disponibles para la venta (o privados) contando con una función de producción para cada uno de los sectores.

En el Cuadro 7 se puede ver los resultados de la estimación de la Ecuación (4) para cada uno de los cinco sectores. Los resultados para el sector Agricultura no se incluyen en el cuadro ya que carecían de sentido económico, hecho que evidencia la escasa asimilación de la operatividad y características propias de este sector con una función de producción como la Cobb-Douglas. Los restantes sectores parecen ajustarse a dicha forma funcional, pues presentan rendimientos constantes a escala en todos los factores y los valores de los parámetros pueden interpretarse económicamente. 


\section{CUADRO 7 \\ CAPITAL PÚB. ECONÓMICO Y PRODUCCIÓN POR SECTORES}

\begin{tabular}{|l|c|c|c|c|}
\hline \multicolumn{1}{|c|}{ Variables } & ENE & CON & IND & SER \\
\hline L & 0,329 & 0,540 & 0,515 & 0,269 \\
& $(2,797)^{* *}$ & $(12,238)^{* *}$ & $(6,934)^{* *}$ & $(5,543)^{* *}$ \\
K & 0,380 & 0,182 & 0,190 & 0,720 \\
& $(2,136)^{* *}$ & $(3,159)^{* *}$ & $(2,493)^{* *}$ & $(7,373)^{* *}$ \\
KGE & $\mathbf{0 , 3 1 8}$ & $\mathbf{- 0 , 0 0 4}$ & $\mathbf{0 , 3 0 7}$ & $\mathbf{- 0 , 0 5 9}$ \\
& $\mathbf{( 1 , 2 0 4 )}$ & $\mathbf{( - 0 , 0 4 0 )}$ & $\mathbf{( 4 , 1 1 1 ) * *}$ & $\mathbf{( - 1 , 0 8 7 )}$ \\
KH & $-0,028$ & 0,112 & $-0,025$ & 0,140 \\
& $(-0,670)$ & $(3,426)^{* *}$ & $(-0,576)$ & $(3,593)^{* *}$ \\
KT & 0,020 & 0,160 & $-0,127$ & $-0,032$ \\
& $(0,153)$ & $(2,878)^{* *}$ & $(-3,745)^{* *}$ & $(-1,230)$ \\
\hline R & 0,979 & 0,997 & 0,998 & 0,999 \\
F & 1085,0 & 8335,2 & 4914,7 & 18417,9 \\
D-W & 2,08 & 1,69 & 2,10 & 1,94 \\
\hline Notas: Los valores entre paréntesis son estadísticos t. * Significatividad al 10\%; ** Significatividad al \\
5\%.
\end{tabular}

Así, los coeficientes de los factores empleo y capital físico privado dieron siempre positivos, significativos al 5\%, aunque su magnitud variaba por sectores. Las elasticidades del $V A B$ con respecto al empleo en los sectores Industria y Construcción sobrepasan el $50 \%$ mientras que las del capital físico privado se sitúan cercanas al $20 \%$. Por su parte, en los sectores de Energía y Servicios Disponibles para la Venta (más en este último) el factor capital físico privado tiene un mayor protagonismo en detrimento del empleo y los demás factores.

La variable central del análisis en este trabajo, el capital público económico, se comporta de una manera un tanto dispar. Para los sectores Energía e Industria se obtuvieron unos valores positivos y considerablemente altos de sus elasticidades, 0,318 y 0,307 respectivamente, aunque únicamente alcanza significatividad estadística el del sector industrial. En el caso de la Construcción y los Servicios las elasticidades obtenidas, aunque son bastante bajas y muestran el signo contrario al esperado carecen de significatividad estadística. En cuanto a los capitales humano y tecnológico, también se obtienen resultados disímiles.

El capital humano es positivo y estadísticamente significativo en los sectores Servicios y Construcción mientras que en Energía e Industria no alcanzan significatividad. El caso del capital tecnológico merece comentarios adicionales. Los valores de sus elasticidades en Energía y Construcción tan sólo son estadísticamente significativo para el segundo, aunque ambos resultan positivos (0,020 y 0,160 respectivamente). A su vez, en los 
sectores de la Industria y los Servicios las elasticidades resultan negativas, aunque sólo sea significativa para el primero de estos sectores.

\section{CUADRO 8 \\ SUBCATEGORÍAS DEL CAPITAL PÚBLICO Y PRODUCCIÓN EN LA INDUSTRIA Y LOS SERVICIOS D.V.}

\begin{tabular}{|c|c|c|c|c|c|c|c|c|}
\hline Variables & 1 & 2 & 3 & 4 & 5 & 6 & 7 & 8 \\
\hline $\mathrm{L}$ & 0,390 & 0,386 & 0,397 & 0,512 & 0,518 & 0,394 & 0,406 & 0,393 \\
\hline K & 0,504 & 0,501 & 0,549 & 0,640 & 0,508 & 0,605 & 0,571 & 0,602 \\
\hline KG & $\begin{array}{c}(4,868)^{* *} \\
\mathbf{0 , 1 0 4} \\
(1,869) *\end{array}$ & $(4,916)^{* *}$ & $(5,659)^{* *}$ & $(5,072)^{* *}$ & $(6,123)^{* *}$ & $(6,230)^{* *}$ & $(6,099)^{* *}$ & $(4,965)^{* *}$ \\
\hline KGE & & $\begin{array}{c}0,107 \\
(2,113) * *\end{array}$ & & & & & & \\
\hline Carretaut & & & $\begin{array}{c}0,053 \\
(1,671)^{*}\end{array}$ & & & & & \\
\hline Ptos & & & & $\begin{array}{c}-0,116 \\
(-1,155)\end{array}$ & & & & \\
\hline Arptos & & & & & $\begin{array}{c}0,008 \\
(0,722)\end{array}$ & & & \\
\hline Ferroc & & & & & & $\begin{array}{c}-0,012 \\
(-0,553)\end{array}$ & & \\
\hline InfHid & & & & & & & $\begin{array}{c}0,062 \\
(1,789) *\end{array}$ & \\
\hline EstUrb & & & & & & & & $\begin{array}{c}-0,003 \\
(-0,122)\end{array}$ \\
\hline $\mathrm{KH}$ & $\begin{array}{c}0,108 \\
(2,228)^{* *}\end{array}$ & $\begin{array}{c}0,109 \\
(2,247)^{* *}\end{array}$ & $\begin{array}{c}0,116 \\
(2,408)^{* *}\end{array}$ & $\begin{array}{c}-0,038 \\
(-0,758)\end{array}$ & $\begin{array}{c}0,003 \\
(0,091)\end{array}$ & $\begin{array}{c}0,122 \\
(2,533)^{* *}\end{array}$ & $\begin{array}{c}0,112 \\
(2,329)^{* *}\end{array}$ & $\begin{array}{c}0,122 \\
(2,509)^{* *}\end{array}$ \\
\hline $\mathrm{KT}$ & $\begin{array}{c}-0,081 \\
(3,229)^{* *}\end{array}$ & $\begin{array}{c}-0,078 \\
(3,105)^{* *}\end{array}$ & $\begin{array}{c}-0,080 \\
(3,204)^{* *}\end{array}$ & $\begin{array}{c}0,029 \\
(0,689)\end{array}$ & $\begin{array}{c}0,004 \\
(0,140)\end{array}$ & $\begin{array}{c}-0,081 \\
(3,149)^{* *}\end{array}$ & $\begin{array}{c}-0,086 \\
(3,466)^{* *}\end{array}$ & $\begin{array}{c}-0,078 \\
(3,096)^{* *}\end{array}$ \\
\hline $\mathrm{R}^{2}$ & 0,999 & 0,999 & 0,999 & 0,999 & 0,999 & 0,999 & 0,999 & 0,999 \\
\hline F & 13427,4 & 13503,6 & 13372,6 & 20572,9 & 20622,4 & 13189,9 & 18290,9 & 13170,9 \\
\hline D-W & 2,02 & 2,02 & 2,02 & 2,10 & 1,98 & 1,99 & 2,16 & 1,99 \\
\hline
\end{tabular}

Una posible explicación del comportamiento de esta variable con la que se mide el capital tecnológico puede encontrarse examinando la regionalización de los gastos en I+D. Analizando los gastos, y a su vez los stocks calculados, por comunidades autónomas se encontró que, de media para el periodo estudiado, en la Comunidad de 
Madrid (43,73\%) y Cataluña (19,14\%) se concentraron casi dos tercios del stock total de capital tecnológico de España. Si se tiene en cuenta a País Vasco $(8,46 \%)$ y Andalucía $(8,01 \%)$ en este cálculo la cifra se aproxima al 80\% entre las cuatro comunidades, dejando a las trece restantes un escaso $20 \% 0^{14}$.

Aunque el ejercicio anterior se aproxima al conocimiento de los factores que determinan la producción sectorial (y entre ellos el capital público), parece que el grado de desagregación de la economía en sus sectores habituales más relevantes resta robustez a los resultados del apartado 4.1. Sin embargo, teniendo en cuenta que dos sectores de la economía española como son Industria y Servicios Disponibles para la Venta han representado de media en los últimos años casi tres cuartas partes del $V A B$ total español, se ha optado por estimar el modelo agregando estos dos sectores.

En las columnas 1 y 2 del Cuadro 8 se parte de la estimación del modelo con capital público total y capital público económico respectivamente para evidenciar los similares comportamientos de las dos modalidades, aunque el coeficiente de KGE es levemente mayor que el de $K G$ y es significativo al $5 \%$, mientras que la última lo es al $10 \%$. Las restantes variables explicativas presentan comportamientos casi idénticos de donde se puede destacar la estabilidad de sus parámetros, incluyendo el resultado del capital tecnológico. En las columnas 3 a 8 se analizan los efectos de las subcategorías económicas. En primera instancia debe señalarse como positiva la estabilidad en los parámetros de los cuatro factores diferentes al capital público, con excepción de las columnas 4 y 5 , debido posiblemente a la no existencia de puertos y aeropuertos en todas las comunidades autónomas.

Las subcategorías del capital público se comportan de forma similar a las de la estimación para la economía agregada. Para el caso de carreteras y autopistas e infraestructuras hidráulicas (columnas 3 y 7) se obtienen unos parámetros positivos (0,053 y 0,062 respectivamente) y significativos lo que demuestra, una vez más, su relevancia en los procesos productivos privados. Por su parte, las otras cuatro categorías no alcanzan significatividad a los niveles habituales.

Combinando algunas de las demás subcategorías junto a carreteras y autopistas e infraestructuras hidráulicas se realizaron estimaciones adicionales cuyos resultados aparecen en el Cuadro 9.

\footnotetext{
14 Además, al estimar el modelo con efectos comunes en vez de fijos el coeficiente de $K T$ resulta positivo y significativo indicando quizá que parte del efecto de esta variable sobre el VAB lo absorben los efectos fijos.
} 


\section{CUADRO 9}

\section{CATEGORIAS CLAVE DEL CAPITAL PÚBLICO Y PRODUCCIÓN EN LA INDUSTRIA Y LOS SERVICIOS DV}

\begin{tabular}{|c|c|c|c|c|}
\hline Variables & 1 & 2 & 3 & 4 \\
\hline \multirow[t]{2}{*}{$\bar{L}$} & 0,400 & 0,391 & 0,388 & 0,390 \\
\hline & $(6,623)^{* *}$ & $(6,478)^{* *}$ & $(6,386)^{* *}$ & $(6,465)^{* *}$ \\
\hline \multirow[t]{2}{*}{ K } & 0,547 & 0,551 & 0,541 & 0,543 \\
\hline & $(5,748) * *$ & $(5,812)^{* *}$ & $(5,568) * *$ & $(5,701)^{* *}$ \\
\hline Carretaut+InfHid+ & 0,089 & & & \\
\hline Ptos & $(2,092) * *$ & & & \\
\hline \multirow{2}{*}{$\begin{array}{l}\text { Carretaut+InfHid+ } \\
\text { Arptos }\end{array}$} & & 0,086 & & \\
\hline & & $(2,142)^{* *}$ & & \\
\hline \multirow{2}{*}{$\begin{array}{l}\text { Carretaut+Ptos }+ \\
\text { Arptos }\end{array}$} & & & 0,064 & \\
\hline & & & $(1,858) *$ & \\
\hline \multirow{2}{*}{$\begin{array}{l}\text { Carretaut+InfHid+ } \\
\text { Ptos+Arptos }\end{array}$} & & & & 0,097 \\
\hline & & & & $(2,236) * *$ \\
\hline \multirow[t]{2}{*}{$\mathrm{KH}$} & 0,109 & 0,107 & 0,114 & 0,107 \\
\hline & $(2,266) * *$ & $(2,211)^{* *}$ & $(2,363) * *$ & $(2,218)^{* *}$ \\
\hline \multirow[t]{2}{*}{$\mathrm{KT}$} & $-0,085$ & $-0,084$ & $-0,079$ & $-0,084$ \\
\hline & $(-3,404) * *$ & $(-3,370)^{* *}$ & $(-3,165)^{* *}$ & $(-3,374)^{* *}$ \\
\hline $\mathrm{R}^{2}$ & 0,999 & 0,999 & 0,999 & 0,999 \\
\hline $\mathrm{F}$ & 13491,6 & 13512,6 & 13424,2 & 13545,6 \\
\hline D-W & 2,03 & 2,03 & 2,02 & 2,03 \\
\hline
\end{tabular}

Las categorías que mostraron mejor comportamiento en este ejercicio fueron puertos y aeropuertos. En la columna 1 se presentan los resultados para uno de los grupos de tres categorías, añadiendo puertos a las dos ya mencionadas. En la columna 2 se hace lo propio con aeropuertos; en la 3 se estiman carreteras y autopistas junto con puertos y aeropuertos, y en la 4 se logra el conjunto más robusto de cuatro categorías, es decir las más productivas.

En suma, tanto en el ámbito agregado como sectorial, los resultados parecen confirmar el importante papel que juega el capital público económico en el nivel de producción de la economía. Queda manifiesto, asimismo, que hay categorías de éste que inciden en mayor grado que otras sobre el VAB regional. 


\section{CONCLUSIONES}

En este trabajo se analiza el impacto del capital público en la producción de las diferentes comunidades autónomas españolas. Deben destacarse tres elementos innovadores en este estudio: en primer lugar, el análisis se realiza tanto a nivel de la economía agregada como de los principales sectores productivos; en segundo lugar, se incorporan dos nuevas variables relevantes, como el capital humano y el capital tecnológico, apenas tenidas en cuenta en trabajos previos con un modelo como este; y por último, se amplía el periodo temporal hasta 2001.

Los resultados obtenidos permiten evidenciar para la economía española la relevancia de las infraestructuras como factor explicativo de variaciones en el output. La contribución de este capital se asemeja a la de otros trabajos con una elasticidad positiva y significativa de 0,156. Pudo comprobarse también la importancia de la composición del capital público, pues las infraestructuras más relacionadas con la actividad productiva (capital público económico) mostraron una elasticidad significativa de 0,138 , mientras que el capital público social aunque presentó un coeficiente negativo no alcanzó significatividad estadística.

La estimación del impacto desagregado del capital público revela que no todas las categorías tienen el mismo efecto sobre la actividad económica, y tres se destacan como las más productivas: carreteras y autopistas, aeropuertos, e infraestructuras hidráulicas. Asimismo, pudo comprobarse la presencia de spillovers o "efectos desbordamiento" en el capital público económico mostrando mayor magnitud relativa en las carreteras y autopistas. El análisis por sectores puso en evidencia que la industria y los servicios disponibles para la venta son más sensibles al efecto del capital público que la agricultura, la energía o la construcción. La elasticidad del producto con respecto al capital público económico para industria y servicios fue de 0,107 y estadísticamente significativa.

Además, pudo hallarse evidencia en contra a lo sostenido en otros estudios, como por ejemplo en Fernández y Polo (2001 y 2002), en referencia a la presencia del capital humano y tecnológico en estos modelos. Los resultados en este trabajo sugieren que el efecto del capital público sobre la productividad privada no es muy sensible a la introducción de otras variables que afecten a ésta como es el caso del capital humano y el capital tecnológico, pues la inclusión de ellas en el modelo estimado apenas altera el coeficiente de la variable KGE, pasando de 0,133 sin $K H$ y $K T$, a 0,138 con KHy KT.

Teniendo en cuenta la imperante necesidad de los gobiernos de optimizar sus recursos de inversión y destinarlos a los usos que prometen mayor productividad, los resultados aquí presentados podrían ser sugerentes. Sin duda, la elasticidad de la producción del sector privado con respecto al stock de capital público, específicamente en forma de 
carreteras y autopistas, aeropuertos, infraestructuras hidráulicas y estructuras urbanas puede ayudar a fundamentar una política eficaz de infraestructuras tendiente, por una parte, a disminuir las desigualdades en las dotaciones de las distintas regiones, y por otra, a potenciar el crecimiento del producto en cada una de ellas y, por ende, en la economía nacional.

\section{REFERENCIAS}

- Álvarez, A., L. Orea y J. Fernández, (2003): "La Productividad de las Infraestructuras en España”. Papeles de Economía Española, No. 95, págs. 125-136.

- Arguimón, I., J. M. González-Páramo, M. J. Martín y J. M. Roldán (1994): “Productividad e Infraestructuras en la Economía Española”. Moneda y Crédito, 198, págs. 207-252.

- Aschauer, D. A. (1989): “Is Public Expenditure Productive?" Journal of Monetary Economics, 23, págs. 177-200.

- Bajo, O. y S. Sosvilla (1993): "Does Public Capital Affect Private Sector Performance? An Análisis of the Spanish Case 1964-1988”. Economic Modelling, 10, págs. 179-185.

- Biehl, D. (1988): "Las Infraestructuras y el Desarrollo Regional". Papeles de Economía Española, 35, págs. 293-310.

- De La Fuente, A. y Vives X. (1995): "Infrastructure and Education as Instruments of Regional Policy: evidence from Spain”. Economic Policy, 20, págs. 13-51.

- Delgado, M. J. y I. Álvarez, (2000): “Las Infraestructuras Productivas en España: estimación del stock en unidades físicas y análisis de su impacto en la producción privada regional”. Revista Asturiana de Economía, 19, págs. 205-231.

- Delgado, M. J. y I. Álvarez, (2004): "Infraestructuras y Eficiencia Técnica: Un Análisis de Técnicas Frontera”. Revista de Economía Aplicada, Vol. XII, No. 35, págs. 65-81.

- Fernández, M. y C. Polo, (2001): “Capital Público y Productividad Privada en España: una panorámica”. Revista Galega de Economía, Vol. 10, 1, págs. 105-132.

- Fernández, M. C. Polo, (2002): "Productividad del Capital Público en Presencia de Capital Tecnológico y Humano". Revista de Economía Aplicada, Vol. X, No. 29, págs. 151-161.

- Freire-Serén, M. J. y J. Alonso. (2002): "Infraestructuras Públicas y Desarrollo Económico de Galicia”. En: De La Fuente, A., M. J. Freire, y J. Alonso, Infraestructuras y Desarrollo Regional, Documentos De Economía 15, Fundación CaixaGalicia, págs. 33-67.

- González Páramo, J. M. (1995): “Infraestructuras, Productividad y Bienestar", Investigaciones Económicas, Vol. XIX, 1, págs. 155-168.

- Gramlich, E. M. (1994): "Infrastructures Investment: A Review Essay". Journal of Economic Literature, Vol. 32, págs. 1176-1196.

- Greene W. H. (1999). “Análisis Econométrico, 3a edición”, Prentice Hall, Madrid, pág. 536.

- Griliches, Z. (1979): "Issues in assessing the contribution of research and development to productivity growth", Bell Journal of Economics, vol. 10, 1, págs. 92-116.

- Hirschman, A. O. (1958): “The Strategy of Economic Development.” New Haven.

- Instituto Nacional de Estadística (2005). "Contabilidad Regional de España". Macromagnitudes Regionales, varias series. En: www.ine.es. 
- Jones, C. I. (2000): “Introducción al Crecimiento Económico”. Pearson Educación. México, págs. 39, 161.

- Mas, M., J. Maudos, F. Pérez y E. Uriel. (1996): "Infrastructures and Productivity in the Spanish Regions”. Regional Studies, Vol. 30 (7), págs. 641 - 649.

- Mas, M., F. Pérez y E. Uriel. (2005a): "El Stock de Capital en España y su Distribución Territorial, (1964-2002)". Fundación BBVA, Bilbao.

- $\quad$ Mas, M., F. Pérez y E. Uriel. (2005b): "El Stock y los Servicios del Capital en España, (19642002), Nueva Metodología”. Fundación BBVA, Bilbao.

- Meade, J. (1952): "External Economies and Diseconomies in a Competitive Situation", Economic Journal, 62, págs. 54-67.

- Munnell, A. (1992): "Infrastructure Investment and Economic Growth". Journal of Economic Perspective, Vol. 4, No. 4, págs. 189-198.

- Pérez, D. y J. Sanaú, (2004): “¿Es el Capital Público Realmente Productivo?”. Comunicación presentada en XXIX Reunión de Estudios Regionales, Asociación Española de Ciencia Regional, págs. 1-22.

- Sanaú, J. (1995): "Unas Notas Sobre la Naturaleza y el Estudio de las Infraestructuras". Cuadernos Aragoneses de Economía, Vol. 5, No. 2, págs. 433-443.

- Sanaú, J. (1997): "La contrastación de la hipótesis del capital público en España: principales aportaciones". Cuadernos Aragoneses de Economía, Vol. 7, No. 2, págs. 281-314.

- Sanaú, J. (1998): "Telecommunications Infrastructure and Economic Growth: An Analysis of Spanish Manufacturing Industry". En: S. Macdonald y G. Madden (Eds.): "Telecommunications and Socio-Economic Development". Elsevier Science B. V., págs. 39-54.

- Sala i Martin, X. (2000): “Apuntes de Crecimiento Económico”. 2da. Edición. Antoni Bosch Editor. Barcelona, pág. 136.

- Soete, L. y P. Patel. (1985): "Recherche-Développement, importations de technologie et croissance économique. Une tentative de comparaison internationales", Revue Économique, vol. 36, 5, págs. 975-1000. 